\title{
REPRESENTING HOMOLOGY CLASSES BY SYMPLECTIC SURFACES
}

\author{
M. J. D. Hamilton
}

Abstract. We derive an obstruction to representing a homology class of a symplectic 4-manifold by an embedded, possibly disconnected, symplectic surface.

A natural question concerning symplectic 4-manifolds is the following: given a closed symplectic 4-manifold $(M, \omega)$ and a homology class $B \in H_{2}(M ; \mathbb{Z})$, determine whether there exists an embedded, possibly disconnected, closed symplectic surface representing the class $B$. This question has been studied by Lê and $\mathrm{Li}[8,9]$. We always assume that the orientation of a symplectic surface is the one induced by the symplectic form. One necessary condition is then, of course, that the symplectic class $[\omega]$ evaluates positively on the class $B$, meaning that $\langle[\omega], B\rangle>0$. Among other things, it is shown in [9] that a class $B$ with $\langle[\omega], B\rangle>0$ in a symplectic 4-manifold is always represented by a symplectic immersion of a connected surface. It is also noted that an obstruction to representing a homology class $B$ by an embedded connected symplectic surface comes from the adjunction formula: the (even) integer

$$
K_{M} B+B^{2}
$$

where $K_{M}$ denotes the canonical class of the symplectic 4-manifold $(M, \omega)$, has to be at least -2 . This obstruction, however, disappears, if the number of components of the symplectic surface is allowed to grow large. Note that there are examples of classes in symplectic 4-manifolds, which are represented by an embedded disconnected symplectic surface, but not by a connected symplectic surface: for example in the twofold blow-up $X \# 2 \overline{\mathbb{C P}}^{2}$ of any closed symplectic 4-manifold $X$ the sum of the classes of the exceptional spheres is not represented by a connected embedded symplectic surface according to the adjunction formula. It is the purpose of this paper to derive an obstruction to representing a homology class by an embedded, possibly disconnected, symplectic surface.

In [9], it is also shown that for symplectic manifolds $M$ of dimension at least six, every class in $H_{2}(M ; \mathbb{Z})$ on which the symplectic class evaluates positively is represented by a connected embedded symplectic surface. In [8], there is a conjecture that in the case of symplectic 4-manifolds $M$ says that if $\alpha$ is a class in $H_{2}(M ; \mathbb{Z})$ on which the symplectic class evaluates positively, then there exists a positive integer $N$ depending on $\alpha$ such that $N \alpha$ is represented by an embedded, not necessarily connected, symplectic surface. In the examples at the end of this paper, we give counterexamples to this conjecture in the four-dimensional case.

Received by the editors August 27, 2012.

2010 Mathematics Subject Classification. Primary 57R17; Secondary 57N13, 57N35.

Key words and phrases. 4-manifold, symplectic, branched covering. 
The non-existence of an embedded symplectic surface in the class $B$ has the following consequence for the Seiberg-Witten invariants, which we only state in the case $b_{2}^{+}>1$.

Proposition 1. Let $(M, \omega)$ be a closed symplectic 4-manifold with $b_{2}^{+}(M)>1$ and $B \neq 0$ an integral second homology class, which cannot be represented by an embedded, possibly disconnected, symplectic surface. Then the Seiberg-Witten invariant of the Spin $^{c}$-structure

$$
s_{0} \otimes P D(B)
$$

is zero, where $s_{0}$ denotes the canonical Spin ${ }^{c}$-structure with determinant line bundle $K_{M}^{-1}$ induced by a compatible almost complex structure.

Here $P D$ denotes the Poincaré dual of a homology class. Note that the first Chern class of the $\operatorname{Spin}^{c}$-structure $s_{0} \otimes P D(B)$ is equal to $-K_{M}+2 P D(B)$. Proposition 1 is a consequence of a theorem of Taubes, relating classes with non-zero Seiberg-Witten invariants to embedded symplectic surfaces [14].

In the following, let $(M, \omega)$ denote a closed symplectic 4-manifold and $\Sigma \subset M$ an embedded, possibly disconnected, closed symplectic surface representing a class $B \in H_{2}(M ; \mathbb{Z})$. We always assume that the orientation of $M$ is given by the symplectic form $(\omega \wedge \omega>0)$. If the class $B$ is divisible by an integer $d>1$, in the sense that there exists a class $A \in H_{2}(M ; \mathbb{Z})$ such that $B=d A$, then there exists a $d$-fold cyclic ramified covering $\phi: \bar{M} \rightarrow M$, branched along $\Sigma$. The branched covering is again a closed symplectic 4-manifold. This is a well-known fact (the pullback of the symplectic form $\omega$ plus $t$ times a Thom form for the preimage $\bar{\Sigma}$ of the branch locus is for small positive $t$ a symplectic form on $\bar{M}$; see $[3,11]$ for a careful discussion). The invariants of $\bar{M}$ are given by the following formulas [4, p. 243], [5]:

$$
\begin{aligned}
K_{\bar{M}} & =\phi^{*}\left(K_{M}+(d-1) P D(A)\right) \\
K_{\bar{M}}^{2} & =d\left(K_{M}+(d-1) P D(A)\right)^{2} \\
w_{2}(\bar{M}) & =\phi^{*}\left(w_{2}(M)+(d-1) P D(A)_{2}\right) \\
\sigma(\bar{M}) & =d\left(\sigma(M)-\frac{d^{2}-1}{3} A^{2}\right)
\end{aligned}
$$

Here $P D(A)_{2} \in H^{2}\left(M ; \mathbb{Z}_{2}\right)$ is the $\bmod 2$ reduction of $P D(A)$. The second equation follows from the first because the branched covering map has degree $d$.

Suppose that the branched covering $\bar{M}$ is symplectically minimal and not a ruled surface over a curve of genus $>1$. Then theorems of Taubes and Liu [10,13] imply that $K_{\bar{M}}^{2} \geq 0$. With the formula above, we obtain the following obstruction on class $A$.

Theorem 2. Let $(M, \omega)$ be a closed symplectic 4-manifold, $\Sigma \subset M$ an embedded, possibly disconnected, closed symplectic surface and $d>1$ an integer such that $d A=$ $[\Sigma]$ for a class $A \in H_{2}(M ; \mathbb{Z})$. Consider the $d$-fold cyclic branched cover $\bar{M}$, branched along $\Sigma$. If $\bar{M}$ is minimal and not a ruled surface over a curve of genus $>1$, then

$$
\left(K_{M}+(d-1) P D(A)\right)^{2} \geq 0 .
$$

It is therefore important to ensure that the branched covering $\bar{M}$ is minimal and not a ruled surface. First, we have the following lemma. 
Lemma 3. Let $\phi: \bar{M} \rightarrow M$ be a cyclic d-fold branched covering of closed oriented 4-manifolds. Then $b_{2}^{+}(\bar{M}) \geq b_{2}^{+}(M)$.

Proof. With our choice of orientations, the map $\phi: \bar{M} \rightarrow M$ has positive degree. By Poincaré duality, the induced map $\phi^{*}: H^{*}(M ; \mathbb{R}) \rightarrow H^{*}(\bar{M} ; \mathbb{R})$ is injective. It maps classes in the second cohomology of positive square to classes of positive square. This implies the claim.

Proposition 4. In the notation of Theorem 2, each of the following two conditions implies that $\bar{M}$ is minimal and has $b_{2}^{+}(\bar{M})>1$ and hence is not a ruled surface:

(a) If $d$ is odd assume that $M$ is spin and if $d$ is even assume that $P D(A)$ is characteristic. Also assume that $3 \sigma(M) \neq\left(d^{2}-1\right) A^{2}$.

(b) Assume that $b_{2}^{+}(M) \geq 2$ and there exists an integer $k \geq 2$ such that the class

$$
K_{M}+(d-1) P D(A)
$$

is divisible by $k$.

Proof. Consider the $d$-fold branched covering $\bar{M}$, branched along $\Sigma$. The assumptions in case (a) imply that $\bar{M}$ is spin and that the signature $\sigma(\bar{M})$ is non-zero. According to a theorem of Furuta [2], we have $b_{2}^{+}(\bar{M}) \geq 3$. Also the symplectic manifold $\bar{M}$ is minimal, because it is spin. In case (b), the lemma implies that $b_{2}^{+}(\bar{M}) \geq 2$. In addition, the symplectic manifold $\bar{M}$ is minimal, because its canonical class is divisible by $k$ (a non-minimal symplectic 4-manifold $Y$ contains a symplectic sphere $S$ with $\left.K_{Y} S=-1\right)$.

Example 5. Consider $M=K 3$. Then we have $K_{M}=0$. Let $d \geq 3$ be an integer and $A \in H_{2}(M ; \mathbb{Z})$ a class with $A^{2}<0$. Theorem 2 together with Proposition 4 part (b) imply that $d A$ is not represented by an embedded symplectic surface. Note that $K 3$ contains indivisible classes of negative self-intersection which, for a suitable choice of symplectic structure, are represented by symplectic surfaces, for example symplectic $(-2)$-spheres. Let $A$ be the homology class of such a sphere and $\alpha=3 A$. Then $\alpha$ is a counterexample to Lê's Conjecture 1.4 in [8].

Example 6. Let $X$ be a closed symplectic spin 4-manifold with $b_{2}^{+}>1$ and $M$ the blow-up $X \# \overline{\mathbb{C P}}^{2}$. Let $E$ denote the class of the exceptional sphere in $M$. We have $K_{M}=K_{X}+P D(E)$. For every positive even integer $d$ with $d^{2}>K_{X}^{2}$, the class $d E$ is not represented by a symplectic surface. Taking for example the blow-up of the $K 3$ surface and $\alpha=2 E$, we obtain another counterexample to Lê's conjecture.

Note that with this method it is impossible to find a counterexample to Lê's conjecture under the additional assumption that $\alpha^{2}>0$.

In light of the second example, the following conjecture seems natural.

Conjecture. Let $M$ be the blow-up $X \# \overline{\mathbb{C P}}^{2}$ of a closed symplectic 4-manifold $X$ and $E$ the class of the exceptional sphere. Then $d E$ is not represented by an embedded symplectic surface for all integers $d \geq 2$.

This conjecture holds by a similar argument as above for $X$ the $K 3$ surface and the 4 -torus $T^{4}$. Moreover, using positivity of intersections, the conjecture holds in the complex category for the blow-up of a complex surface and embedded complex curves. In fact, in this category the result holds not only for the exceptional curve in 
a blow-up, but for multiples of the class of any connected embedded complex curve with negative self-intersection in a complex surface.

Remark 7. Branched covering arguments have been used in the past to find lower bounds on the genus of a connected surface representing a divisible homology class in a closed 4-manifold; see $[1,6,7,12]$.

\section{Acknowledgment}

I would like to thank D. Kotschick for very helpful comments.

\section{References}

[1] J. Bryan, Seiberg-Witten à la Furuta and genus bounds for classes with divisibility, Turkish J. Math. 21 (1997), 55-59.

[2] M. Furuta, Monopole equation and the $\frac{11}{8}$-conjecture, Math. Res. Lett. 8(3) (2001), 279-291.

[3] R.E. Gompf, Symplectically aspherical manifolds with non-trivial $\pi_{2}$, Math. Res. Lett. 5(5), (1998), 599-603.

[4] R.E. Gompf and A.I. Stipsicz, 4-manifolds and Kirby calculus, number 20 in Graduate Studies in Mathematics, American Mathematical Society, 1999.

[5] F. Hirzebruch, The signature of ramified coverings, in Global analysis (Papers in Honor of K. Kodaira), 253-265, University of Tokyo Press, 1969.

[6] W.C. Hsiang and R.H. Szczarba, On embedding surfaces in four-manifolds, Proc. Symp. Pure Math. 22 (1971), 97-103.

[7] D. Kotschick and G. Matić, Embedded surfaces in four-manifolds, branched covers, and $\mathrm{SO}(3)$ invariants, Math. Proc. Cambridge Philos. Soc. 117 (1995), 275-286.

[8] H.-V. Lê, Realizing homology classes by symplectic submanifolds, Preprint 61, Max-PlanckInstitute for Mathematics in the Sciences, 2004.

[9] T.-J. Li, Existence of symplectic surfaces, in Geometry and topology of manifolds, Vol. 47 of Fields Inst. Commun., 203-217, American Mathematical Society, 2005.

[10] A.-K. Liu, Some new applications of general wall crossing formula, Gompf's conjecture and its applications, Math. Res. Lett. 3(5) (1996), 569-585.

[11] F. Pasquotto, On the geography of symplectic manifolds, $\mathrm{PhD}$ thesis, Leiden University, 2004.

[12] V.A. Rohlin, Two-dimensional submanifolds of four-dimensional manifolds, Func. Analysis Appl. 6 (1971), 39-48.

[13] C.H. Taubes, The Seiberg-Witten and Gromov invariants, Math. Res. Lett. 2(2) (1995), 221-238.

$[14] \longrightarrow, S W \Rightarrow G r$ : from the Seiberg-Witten equations to pseudo-holomorphic curves, J. Amer. Math. Soc. 9 (1996), 845-918.

Fachbereich Mathematik, Universität Stuttgart, Pfaffenwaldring 57, 70569 Stuttgart, Germany

E-mail address: mark.hamilton@math.lmu.de 